ARTÍCULO ORIGINAL

\title{
Análisis de isonimia entre poblaciones del noroeste de Colombia
}

\author{
Gabriel Bedoya ${ }^{1}$, Jenny García ${ }^{2}$, Patricia Montoya ${ }^{2}$, Winston Rojas ${ }^{1}$, Maria Eugenia \\ Amézquita ${ }^{3}$, Iván Soto ${ }^{1}$, Maria Cecilia López ${ }^{2}$, Jorge Ospina-Duque ${ }^{2}$, Andrés Ruiz-Linares ${ }^{1}$ \\ ${ }^{1}$ Grupo de Genética Molecular (GENMOL), Facultad de Medicina, Universidad de Antioquia, Medellín, \\ Colombia. \\ ${ }^{2}$ Grupo de Investigación en Psiquiatría (GIPSI), Departamento de Psiquiatría, Facultad de Medicina, \\ Universidad de Antioquia, Medellín, Colombia. \\ ${ }^{3}$ Departamento de Psiquiatría, Universidad de Caldas, Manizales, Colombia.
}

Introducción. La utilización de la frecuencia de apellidos como marcadores de linajes paternos ha permitido caracterizar poblaciones. Los principios de isonimia se han empleado para determinar el grado de estructuración genética, las tasas de migraciones y las relaciones de ancestría y origen entre poblaciones. Este análisis se aplicó a dos poblaciones históricamente relacionadas y consideradas como aislados genéticos.

Objetivo. Evaluar las relaciones genéticas y de origen entre Aranzazu y Marinilla y su zona de influencia por medio de análisis de frecuencia de apellidos.

Materiales y métodos. A partir de la base de datos del Sistema de Identificación de Beneficiarios de los Programas Sociales, Sisbén, se calcularon los parámetros poblacionales de coeficiente de parentesco $\left(\phi_{\mathrm{ij}}\right)$, la homogeneidad poblacional con los estimadores B (porcentaje de la población que comparte los siete apellidos más frecuentes) y C (15 apellidos más frecuentes) y la distancia genética de Cavalli-Sforza en tres poblaciones del núcleo fundador de Marinilla y Rionegro como población externa.

Resultados. Marinilla y Aranzazu, al igual que las poblaciones de Marinilla y su zona de influencia, mostraron los mayores valores de homogeneidad (valores $B$ entre 0,25 y 0,5 ) comparados con Rionegro $(B=0,159)$ y también mayores valores de parentesco intrapoblacional (valores $\phi_{\mathrm{ii}}$ entre 0,0034 y 0,01 ). Las menores distancias se encontraron entre Marinilla y Aranzazu.

Conclusiones. Aranzazu es una población con características similares a las de Marinilla y su zona de influencia y debido al efecto fundador, estas poblaciones pueden presentar características genéticas similares. Por lo tanto, las enfermedades genéticas, principalmente las de herencia compleja, podrían tener la misma etiología genética en ambas poblaciones, lo que garantizaría las condiciones óptimas para estudios de cartografía genética.

Palabras clave: nombres, genética, población, consanguinidad, efecto fundador, Colombia.

Isonymy analysis between 2 populations in northwestern Colombia.

Introduction. Surname frequency (isonymy) is used as a marker of paternal lineage and is used to characterize human population structure. Principles of isonymy were used to determine the genetic structure, migration rates, ancestry relations and origins of populations. This analysis was applied to two historically related local populations which currently are considered to be genetically isolated.

Objective. The genetic relationships and influence zones of the Aranzazu and Marinilla populations were assessed by means of surname frequency analysis.

Materials and methods. Data originated from database with the title "System of Identification of Beneficiaries of the Social Programs" database or Sisben. Population parameters such as a priori kinship $\left(\phi_{\mathrm{ii}}\right)$, population homogeneity with $\mathrm{B}$ and $\mathrm{C}$ estimators, and Cavalli-Sforza's genetic distance were calculated for (a) three towns of Marinilla and its influence zone and (b) Aranzazu. The Rionegro population served as an external, comparison population. 
Results. The Aranzazu and Marinilla populations showed the higher homogeneity (B value between 0.25 and 0.5$)$ in contrast with Rionegro $(B=0.159)$, as well as greater a priori kinship values ( $\phi_{\mathrm{ij}}$ between 0.003 and 0.010$)$. The lowest distances were found between Marinilla and Aranzazu.

Conclusions. Aranzazu is a population with characteristics similar to those of Marinilla and its influence zone. The close similarity of genetic characteristics for these populations is due probably to a founder effect. Furthermore, the genetic similarity predicts that genetic diseases will have the same etiology in both populations and provides optimum conditions for gene mapping studies.

Key words: Names, genetics, population, consanguinity, founder effect, Colombia

El poblamiento de Antioquia se realizó principalmente a partir de dos nucleos, Santa Fe de Antioquia al occidente y Marinilla al oriente, fundadas en 1541 y 1690 , respectivamente (1). El primer núcleo, incentivado por el desarrollo económico predominantemente minero del siglo XVII, fundó en 1675 a San Lorenzo de Aburrá (Medellín), de donde partió la colonización del suroeste y parte del oriente antioqueño, el cual se convirtió en otro centro de colonización para el suroeste de Antioquia y del Viejo Caldas. El segundo núcleo partió de Marinilla, ubicada a 6 $\mathrm{km}$ de Rionegro, fue poblada por gentes provenientes de Mariquita (actual departamento del Tolima), ciudad de la que dependió hasta 1756 cuando pasó a ser parte de la provincia de Antioquia $(2,3)$. Dada la carencia de grandes minas, se consolidó allí la formación de una cultura agrícola que consideraba la tierra como la única fuente de riqueza y poder, lo cual impulsó a la población a expandirse y fundar los siguientes municipios: El Santuario, El Peñol, Carmen de Viboral, Granada, Cocorná y Guatapé $(2,4)$. Este grupo de municipios lo hemos denominado Marinilla y su zona de influencia. Estudios de demografía genética con marcadores de ADN de los municipios de Marinilla y su zona de influencia demuestran que tienen características genéticas muy semejantes en cuanto al grado de mezcla genética, linajes maternos y paternos y baja diversidad de apellidos fundadores, lo cual permite deducir que

\section{Correspondencia:}

Gabriel Bedoya, Grupo de Genética Molecular, Sede de Investigación Universitaria, Universidad de Antioquia, Cra 62 \#52-59 Laboratorio 430, Medellín, Colombia. Teléfono: 2106466.

jegava@epm.net.co, gbedoya@catios.udea.edu.co

Recibido: 22/06/06; aceptado: 11/10/06 esta zona ha sufrido un alto grado de aislamiento despues de la época colonial y que su crecimiento ha sido por expansión interna más que por migración, fénomeno que sucede con la poblacíon antioqueña en general (5). En los siglos XVIII y $X I X$ familias enteras migraron a la región del Viejo Caldas e intervinieron, junto con descedientes del núcleo de Santafé, en la fundación de algunos pueblos de esta regíon, entre ellos Aranzazu. Este municipio fue fundado en 1853 principalmente por personas originarias de Marinilla, de tal forma que su censo de 1864 compartía 10 de los 15 apellidos principales con los del censo de Marinilla de 1851, momento en que estaba en plena actividad la oleada migratoria de Marinilla y su zona de influencia hacia la región del viejo Caldas $(6,7)$.

Algunos trabajos sobre genética de enfermedades humanas en Marinilla y su zona de influencia han detectado varias entidades mendelianas con patrón de herencia recesiva, como fibrosis quística, albinismo y paladar hendido, y otras con patrones complejos como el trastorno afectivo bipolar (8-10). Se postula que la incidencia de estas enfermedades son el resultado de un efecto fundador y de la práctica de matrimonios consanguíneos producida por el aislamiento poscolonial de la región. Al observar los registros del Hospital Universitario de Caldas se detectaron 38 familias con varios miembros afectados, 28 de las cuales tenían apellidos que estaban entre los 15 más frecuentes de Marinilla. Además, en Marinilla, 39 de las 49 familias identificadas con agregación de trastorno afectivo bipolar poseían apellidos de los 15 más frecuentes de Aranzazu (datos no publicados). Esta observación permite postular que esta y otras enfermedades con agregación familiar que segregan en Aranzazu podrían haber sufrido de un efecto fundador similar 
y que, por ende, el comportamiento de las enfermedades en las dos poblaciones debe ser el mismo, lo cual permitiría poner en práctica estrategias de localización de genes involucrados en estas entidades, aumentando la muestra con familias y asumiendo homogeneidad genética de las entidades en ambas poblaciones (de locus y alélica). Un primer acercamiento para someter a prueba tal postulado es el análisis de isonimia, método creado por Crow y Manage en 1965, quienes utilizaron la frecuencia de apellidos idénticos por parejas para calcular coeficientes de parentesco $(11,12)$. En la actualidad, el estudio comparativo de la frecuencia de apellidos es un método que se puede utilizar no solamente para evaluar consanguinidad en una población (probabilidad de hijos autozigotos) sino que permite determinar estructuración genética entre poblaciones (grado de aislamiento), distancias genéticas y migración en la historia de su crecimiento demográfico. En las culturas hispánicas, el primer apellido se hereda del padre y el segundo de la madre, de tal forma que el primer apellido se transmite de padres a hijos varones como si fuera un marcador molecular en la región no recombinante del cromosoma $Y$, en tanto que en las hijas se pierde este primer apellido. Los estudios poblacionales que utilizan frecuencias de apellidos tienen una gran ventaja sobre los marcadores moleculares del cromosoma $Y$ si se tiene en cuenta la posibilidad de reunir información de bases de datos grandes en diferentes periodos históricos haciendo uso de registros de censos y listas de apellidos en diferentes épocas, lo cual no puede hacerse con marcadores moleculares, pues los estudios de muestras grandes son muy costosos y los datos históricos inasequibles (13). En el presente estudio se utilizaron los apellidos de la base de datos del Sistema de Identificación de Beneficiarios de los Programas Sociales, Sisbén, de los municipios del Carmen de Viboral, El Santuario y Marinilla en el departamento de Antioquia, pertenecientes al núcleo fundador de Marinilla y su zona de influencia, y de Rionegro, considerada como una población derivada del núcleo fundador de Santafé de Antioquia, para realizar comparaciones con los apellidos del municipio de Aranzazu (objeto del estudio), el cual pertenece al departamento de
Caldas, con el fin de probar la hipótesis de que Aranzazu pertenece al núcleo fundador de Marinilla y su zona de influencia. La similitud de apellidos entre Aranzazu y Marinilla debe ser mayor que la encontrada entre Marinilla y Rionegro, ésta última población localizada a sólo 7 km de la primera.

\section{Materiales y métodos}

Se recopilaron los datos del primer apellido de cada uno de los hombres de la base de datos del Sisbén. El tamaño de la muestra fue de de 19.284 individuos de Marinilla, 16.055 del Carmen de Viboral, 11.220 del Santuario, 31.344 de Rionegro y 7.514 de Aranzazu. Se calculó el coeficiente de parentesco intrapoblacional a priori no sesgado por matrimonios al azar $\left(\phi_{\mathrm{ii}}\right)$, el cual se relaciona con el $F_{\text {st }}$ de Wright 1951(14) mediante la ecuación $\mathrm{F}_{\mathrm{st}}=\Sigma \mathrm{w}_{\mathrm{i}} \phi_{\mathrm{ii}}$, es decir, el $\mathrm{F}_{\mathrm{st}}$ es la media ponderada de los coeficientes de parentesco a priori de las poblaciones en estudio y es inverso a la riqueza de apellidos (15). El valor de $\phi_{\mathrm{ii}}$ se obtuvo a partir de la ecuación de Crow y Mange $\left(\phi_{\mathrm{ii}}=\mathrm{I}_{\mathrm{ii}} / 4\right)$ y el estimador $\mathrm{I}_{\mathrm{ii}}$ (isonimia no sesgada debida a matrimonios al azar) se obtuvo por medio de la función $\mathrm{I}_{\mathrm{ii}}=\Sigma_{\mathrm{k}} \mathrm{p}_{\mathrm{ik}}{ }^{2}-1 / \mathrm{N}_{\mathrm{i}}$ propuesta por Morton en 1973 (16) y modificada por Rodríguez-Larralde et al. en 1993 (17), en la cual $p_{\mathrm{ik}}$ es la frecuencia del apellido $\mathrm{k}$ en la población i, y $\mathrm{N}_{\mathrm{i}}$ el número total de apellidos en la muestra $(12,16,17)$. Para determinar el grado de diversidad de apellidos se determino el valor $\mathrm{D}=$ número de apellidos/número total de individuos; además, se calculó para cada población el porcentaje de individuos que comparten los 7 y 15 apellidos más frecuentes (estimadores B y C, respectivamente) $(18,19)$. Un valor alto de B o C es característico de poblaciones aisladas en las que unos pocos apellidos se repiten en un alto porcentaje de la población.

Como medida de distancia genética se determinó el estimativo $\mathrm{f}_{\theta}$ con la ecuación 11,5 de CavalliSforza y Bodmer, 1981 (11); éste se basa en la distancia de la cuerda simplificada para un solo locus y asume cada apellido como un alelo; dicho estimativo representa cuatro veces el cuadrado de la distancia genética $\mathrm{d}$, promediada para $\mathrm{k}$ alelos y $f_{\theta}=4(1-\cos \theta) / k-1$, donde $\cos \theta=\Sigma \sqrt{ } p_{i k} p_{j k}$ representa la sumatoria de la raíz cuadrada del 
producto de la frecuencia del apellido $k$ en las poblaciones i y j para $k$ apellidos. Con la matriz de distancias $\mathrm{f}_{\theta}$ se construyó un árbol para determinar las relaciones de similitud entre las poblaciones mediante el método de Neighbor-Joining $(\mathrm{N}-\mathrm{J})$. Este procedimiento se efectuó con el paquete Phylip 3.57c (20). Adicionalmente, se calculó el porcentaje de apellidos compartidos entre poblaciones.

\section{Resultados}

El número total de apellidos en las cinco poblaciones analizadas fue de 857 , con una diversidad $D=0,0097$ (datos no mostrados), lo cual concuerda con la baja variabilidad de apellidos en la población antioqueña como resultado del aislamiento durante la época colonial. El cuadro 1 contiene los 15 apellidos más frecuentes en cada una de las cinco poblaciones y la medida de diversidad D. El cuadro 2 muestra los estimativos de diversidad $\mathrm{B}$ y $\mathrm{C}$, así como el coeficiente de parentesco intrapoblacional. Las poblaciones de El Santuario y Rionegro muestran el mayor y menor valor de aislamiento, respectivamente (representados por los estimativos B y C). Sin embargo, el rango de $C$ entre 0,269 y 0,662 confirma la poca diversidad de apellidos en la población de Antioquia y específicamente en Marinilla y su zona de influencia (5), característica propia de poblaciones aisladas como lle de la Madeleine, un aislado de la población franco canadiense donde $C$ tiene un valor de 0,42 (19). El menor coeficiente de parentesco $\phi_{\mathrm{ii}}$ se encontró en Rionegro $(0,00259)$ y el mayor en Santuario $(0,01)$. Este estimador de la frecuencia de matrimonios consanguíneos se correlacionó positivamente con los estimadores de diversidad C y B.

En el cuadro 3 se muestran las distancias $f_{\theta}$ y el porcentaje de apellidos compartidos. La menor distancia se encontró entre Aranzazu y Marinilla $(0,00019)$ y la mayor entre Rionegro y Marinilla $(0,00482)$. Sin embargo, la distancia entre Aranzazu y Rionegro $(0,00098)$ resultó menor que las de Aranzazu con El Carmen y con Santuario (0,00192 y 0,00225, respectivamente); la topología del árbol construido a partir de las distancias $f_{\theta}$

Cuadro 1. Los 15 apellidos más frecuentes en las cinco poblaciones.

\begin{tabular}{|c|c|c|c|c|c|c|c|c|c|}
\hline \multicolumn{2}{|c|}{$\begin{array}{c}\text { Marinilla } \\
N=19.284 \\
n=402 \\
D=0,021\end{array}$} & \multicolumn{2}{|c|}{$\begin{array}{c}\text { Aranzazu } \\
N=7.504 \\
n=272 \\
D=0,036\end{array}$} & \multicolumn{2}{|c|}{$\begin{array}{c}\text { Carmen } \\
N=16.055 \\
n=315 \\
D=0,020\end{array}$} & \multicolumn{2}{|c|}{$\begin{array}{c}\text { Santuario } \\
N=11.220 \\
n=268 \\
D=0,024\end{array}$} & \multicolumn{2}{|c|}{$\begin{array}{c}\text { Rionegro } \\
N=31.344 \\
n=666 \\
D=0,021\end{array}$} \\
\hline Apellido & $\mathbf{F}$ & Apellido & $\mathbf{F}$ & Apellido & $\mathbf{F}$ & Apellido & $\mathbf{F}$ & Apellido & $\mathbf{F}$ \\
\hline Giraldo & 0,079 & Salazar & 0,053 & Quintero & 0,068 & Zuluaga & 0,096 & García & 0,048 \\
\hline Gómez & 0,061 & Giraldo & 0,051 & Gómez & 0,046 & Ramírez & 0,089 & Gómez & 0,033 \\
\hline Duque & 0,047 & Gómez & 0,040 & García & 0,042 & Giraldo & 0,086 & Ramírez & 0,027 \\
\hline Ramírez & 0,046 & Martínez & 0,029 & Giraldo & 0,039 & Gómez & 0,083 & Ospina & 0,022 \\
\hline García & 0,037 & López & 0,028 & Zuluaga & 0,038 & Quintero & 0,051 & López & 0,021 \\
\hline Zuluaga & 0,035 & González & 0,027 & Ramírez & 0,034 & Duque & 0,049 & Cardona & 0,020 \\
\hline Castaño & 0,026 & García & 0,023 & Alzate & 0,032 & Aristizábal & 0,047 & Henao & 0,018 \\
\hline Cardona & 0,024 & Arias & 0,022 & Valencia & 0,030 & Salazar & 0,037 & Rendón & 0,018 \\
\hline Quintero & 0,022 & Ocampo & 0,021 & Cardona & 0,028 & Castaño & 0,023 & Sánchez & 0,018 \\
\hline Montoya & 0,018 & Castaño & 0,020 & Osorio & 0,028 & García & 0,019 & Alzate & 0,017 \\
\hline Marín & 0,018 & Zuluaga & 0,019 & Castaño & 0,027 & Jiménez & 0,019 & Giraldo & 0,016 \\
\hline Soto & 0,017 & Morales & 0,018 & Hernández & 0,023 & Soto & 0,017 & Echeverry & 0,016 \\
\hline Serna & 0,017 & Cardona & 0,017 & Gallego & 0,022 & Alzate & 0,016 & Castaño & 0,014 \\
\hline González & 0,016 & Alzate & 0,017 & Arbeláez & 0,021 & Gallego & 0,016 & Montoya & 0,014 \\
\hline López & 0,016 & Marín & 0,016 & González & 0,018 & Montoya & 0,015 & Castro & 0,014 \\
\hline
\end{tabular}

$\mathrm{N}$ es el tamaño de muestra (hombres de la lista del Sisbén)

$\mathrm{n}$ es el número de apellidos diferentes en cada población

$\mathrm{D}$ es la diversidad de apellidos calculada como $\mathrm{n} / \mathrm{N}$.

$F=$ frecuencia 
Cuadro 2. Estimativos de diversidad y coeficiente de parentesco intrapoblacional.

\begin{tabular}{lccc}
\hline Población & $\mathbf{B}$ & $\mathbf{C}$ & $\phi_{\mathrm{ii}}$ \\
\hline Marinilla & 0,333 & 0,481 & 0,0053 \\
Aranzazu & 0,251 & 0,402 & 0,0034 \\
Carmen & 0,299 & 0,496 & 0,0050 \\
Santuario & 0,500 & 0,662 & 0,0100 \\
Rionegro & 0,159 & 0,269 & 0,0025 \\
\hline
\end{tabular}

B es el porcentaje de cada población que comparte los siete apellidos mas frecuentes.

C es el que comparte los 15 más frecuentes.

$\phi_{\mathrm{ii}}$ es el coeficiente de parentesco intrapoblacional.

(figura 1) definió dos parejas de "vecinoscercanos", conformadas por Marinilla-Aranzazu y El Carmen-Rionegro. La población del Santuario se situó a una distancia equidistante entre las dos parejas. El porcentaje de apellidos compartidos entre poblaciones presentó un rango de 31,4\% entre Aranzazu y Rionegro a 52,2\% entre Marinilla y El Carmen; Marinilla y Aranzazu comparten $45,6 \%$ de sus apellidos, semejante al que comparte Marinilla con los otros dos miembros de su zona de influencia.

Marinilla, Aranzazu y Rionegro tienen cinco apellidos en común (Giraldo, Gómez, García, Castaño y Cardona) entre los 15 más frecuentes. Además, los apellidos Gómez y García están entre los siete más frecuentes (cuadro 1); sin embargo, Marinilla y Aranzazu comparten además el apellido Giraldo, el cual no está entre los siete más frecuentes de Rionegro. El apellido Giraldo en Marinilla tiene la frecuencia modal (7,9\%), pero la frecuencia más alta se encuentra en Santuario $(8,6 \%)$, que es la población más cercana a Marinilla. Este apellido es el segundo en frecuencia en Aranzazu (5,1\%), a sólo $0,2 \%$ de

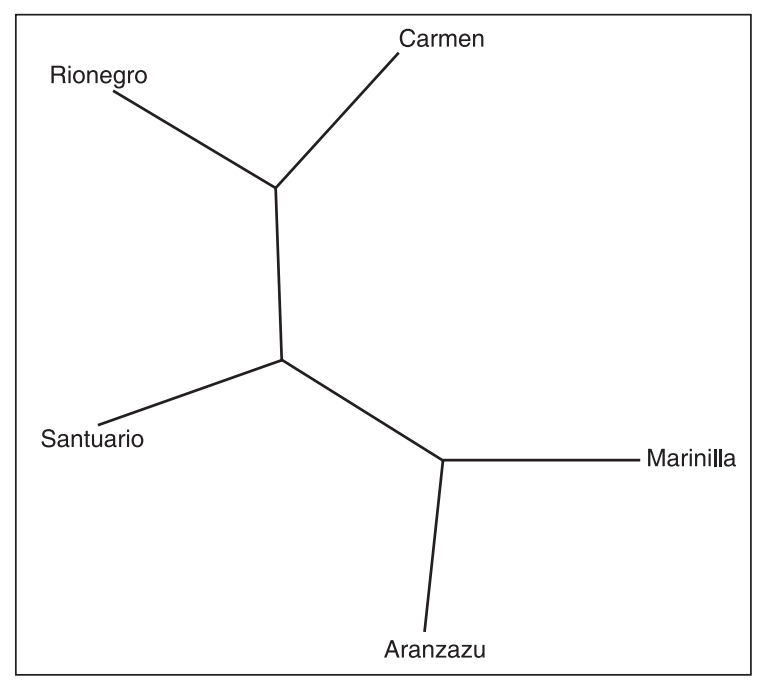

Figura 1. Árbol NJ construido a partir de la matriz de distancias $d_{C E}$ (cuadro 2). Las parejas de vecinos más cercanos son Marinilla-Aranzazu y Rionegro-El Carmen; el Santuario es equidistante a las parejas de $\mathrm{NJ}$

Salazar, el apellido modal. Marinilla y Aranzazu comparten nueve de los 15 apellidos más frecuentes, los cuales representan 31,2 y $24,1 \%$ en cada población, respectivamente. El apellido Zuluaga se encontró en todas las poblaciones, excepto en Rionegro.

\section{Discusión}

La diversidad de los apellidos inferida con los parámetros $\mathrm{B}$ y $\mathrm{C}$ es un indicativo del bajo número de apellidos fundadores en las poblaciones estudiadas y de la dinámica del crecimiento, que debe haberse realizado de manera interna, sin tasas mayores de inmigración después de su fundación. En estas poblaciones se evidencia el efecto de la deriva genética; en particular, Marinilla

Cuadro 3. Porcentaje de apellidos compartidos y distancia genética.

\begin{tabular}{lccccc}
\hline & Marinilla & Aranzazu & Carmen & Santuario & Rionegro \\
\hline Marinilla & $\mathrm{x}$ & 0,00019 & 0,00117 & 0,00104 & 0,00482 \\
Aranzazu & 45,6 & $\mathrm{x}$ & 0,00192 & 0,00225 & 0,00098 \\
Carmen & 52,2 & 44,6 & $\mathrm{x}$ & 0,00189 & 0,00099 \\
Santuario & 48,2 & 44,0 & 49,9 & $\mathrm{x}$ & 0,00148 \\
Rionegro & 44,5 & 31,4 & 38,6 & 33,4 & $\mathrm{x}$ \\
\hline
\end{tabular}

En la parte inferior de la diagonal está la matriz del porcentaje de apellidos que comparten las poblaciones y en la superior, la distancia genética $\mathrm{f}_{\theta}$ entre pares de poblaciones. 
y su zona de influencia y Aranzazu se comportan como aislados genéticos, característica general de la población antioqueña evidenciada con marcadores moleculares $(21,22)$. Se ha demostrado que el uso de apellidos para determinar estructuración entre poblaciones correlaciona mejor con marcadores moleculares cuando se aplica a poblaciones con bajo número de habitantes y que presentan aislamiento (23). Por lo tanto, los datos obtenidos con los parámetros $B$ y $C$ presentan un alto grado de fiabilidad, a pesar de que el valor de $C$ haya sido tan alto en Marinilla y su zona de influencia, incluida Aranzazu, pues el promedio en las cuatro poblaciones $(51 \%)$ es mayor que el encontrado en una de las poblaciones consideradas con el menor grado de flujo génico, como es la población franco-canadiense de lle de la Madeleine (19). Las poblaciones de Marinilla y su zona de influencia, pese a encontrarse cerca de la población de Rionegro, mostraron mayor similitud con la población de Aranzazu, localizada en otro departamento, lo cual es apreciable tanto por la magnitud de los valores de los estimativos $B$ y $C$ como por la composición de apellidos; Aranzazu es muy semejante a Marinilla y su zona de influencia, específicamente a Marinilla. Esto tiene un fuerte sustento en los datos de distancia genética, ya que el comportamiento de $f_{\theta}$ se correlaciona muy bien con los valores de $\mathrm{B}$ y $\mathrm{C}$ (datos no mostrados). Si se tiene en cuenta que esta distancia se calcula bajo el modelo que toma los apellidos como alelos de un locus en el cromosoma $\mathrm{Y}, \mathrm{f}_{\theta}$ es un buen estimador de las diferencias genéticas entre las poblaciones, de tal forma que, aunque las distancias entre las cinco poblaciones presentan valores muy bajos, lo cual demuestra de nuevo homogeneidad genética de la población paisa (5), la distancia Marinilla-Aranzazu $\left(1,9 \times 10^{-4}\right)$ no sólo es la menor de las 10 calculadas, sino que muestra un orden de magnitud menor que la de Marinilla- Rionegro $\left(4,82 \times 10^{-3}\right)$. La topología del árbol $\mathrm{N}-\mathrm{J}$ construido a partir de la matriz de los valores $f_{\theta}$ corrobora la similitud genética entre Marinilla y Aranzazu, puesto que fueron agrupados como vecinos más cercanos por encima de poblaciones pertenecientes a Marinilla y su zona de influencia. Esto se ve reforzado por el porcentaje de apellidos compartidos entre estas poblaciones (45,6\%), levemente menor que el que comparte Marinilla con Santuario, situado sólo a $8 \mathrm{~km}$. De otro lado, la agrupación de El Carmen con Rionegro es un indicio de la migración reciente de pobladores de Rionegro por el auge de la industria de la cerámica en El Carmen.

El origen común de Marinilla y Aranzazu se reafirma al analizar la dinámica de los apellidos más frecuentes entre las poblaciones. Aranzazu comparte ocho de los 15 apellidos más frecuentes y cuatro de los siete más frecuentes de acuerdo al primer censo de Marinilla de 1786, y estas proporciones se mantienen al comparar el primer censo de Aranzazu, realizado en 1864, con el de Marinilla de 1851, fechas muy cercanas a la fundación de Aranzazu (5). La correlación entre los resultados hallados y los sustentos históricos permite determinar que el apellido Giraldo, considerado como uno de los fundadores de Marinilla, pues Francisco Manzueto Giraldo fue una de las cabezas de las 19 familias registradas en el primer padrón de esta fundación en 1670 (3), se encontró en Aranzazu con una frecuencia muy cercana al modal $(5,1 \%)$ en la muestra del Sisbén, y es el modal en la muestra aleatoria (7,1\%). De la misma manera, el apellido Gómez, que en Aranzazu tiene una frecuencia de $4 \%$, es considerado como fundador en Marinilla y su frecuencia de $7 \%$ en el censo de 1786 se ha mantenido constante en esta población hasta el presente. En cuanto al apellido Salazar, que parece tener la misma frecuencia que Giraldo en Aranzazu y es el modal en la muestra del Sisbén, aparece en el censo de Marinilla de 1786 en cuarto lugar, con una frecuencia de $5 \%$. Sin embargo, este apellido actualmente no se encuentra entre los 15 más frecuentes de Marinilla, pero sí en Santuario, con 3,7\%. Por lo tanto, los portadores de este apellido deben haber emigrado de Marinilla donde fue uno de los apellidos fundadores. Esto concuerda con datos históricos sobre la colonización del Viejo Caldas que narran sobre dos concesiones importantes que extendieron la influencia de la población paisa en el sur de Antioquia: la de Villegas y la de Aranzazu; para esta última, propiciada por Juan de Dios de Aranzazu entre 1820 y 1850, eran comunes los 
nombres de Elías González y "Salazar y Compañía" (24-26).

Los resultados de este estudio sugieren que Aranzazu es una población que posee características genéticas semejantes a las poblaciones del núcleo fundador de Marinilla y su zona de influencia. Es posible también que las variantes génicas que circulan en Aranzazu sean las mismas que las que circulan en Marinilla si se tiene en cuenta la alta correlación de los estudios con apellidos y marcadores moleculares del cromosoma $Y(5,23,27-29)$. Por tanto, las enfermedades genéticas, principalmente las de herencia compleja detectadas en Aranzazu, podrían tener igual etiología genética que las encontradas en Marinilla. Entre las estrategias para identificar las variantes génicas involucradas en estas entidades puede pensarse en tomar las familias identificadas en Marinilla y su zona de influencia y Aranzazu, ya sea extendidas (para análisis de ligamiento paramétrico) o nucleares (para estudios de asociación familiar), como una sola muestra, sin correr el riesgo de estratificación, ya que este estudio sugiere que no existe heterogeneidad genética.

\section{Agradecimientos}

Se agradece a los Hospitales San Vicente de Paúl de Aranzazu y San Juan de Dios de Marinilla, a las administraciones municipales y parroquias de Marinilla y Aranzazu.

\section{Conflicto de intereses}

Los autores declaramos que no existe ningún conflicto de intereses.

\section{Financiación}

Este estudio se desarrolló como parte del proyecto 11150412976 financiado por Colciencias.

\section{Referencias}

1. Álvarez V. Poblamiento y población en el Valle de Aburrá y Medellín. En: Melo JO, editor. Historia de Medellín. Medellín: Suramericana; 1996. p.1541-951.

2. Parsons JJ. Antioquian colonization in Western Colombia. 2 ed. Berkeley: University of California Press; 1968.

3. Zapata-Cuéncar H. Monografías de Antioquia. Medellín: Cervunión; 1941.
4. Cornare-Iner. Granada. Medellín: Cornare; 1990.

5. Bedoya G, Montoya P, García J, Soto I, Bourgeois S, Carvajal L, et al. Admixture dynamics in Hispanics: a shift in the nuclear genetic ancestry of a South American population isolate. Proc Natl Acad Sci U S A 2006;103:7234-9.

6. Arango-Mejía G. Genealogías de Antioquia y Caldas. Medellín: Litoarte; 1993.

7. Soto I. Evaluación histórica y genética del origen de Marinilla y su zona de influencia. Medellín, Universidad de Antioquia; 2003.

8. Arcos-Burgos M, Muenke M. Genetics of population isolates. Clin Genet 2002;61:233-47.

9. Ospina J, Duque C, Carvajal L, Ortiz D, Soto I, Pineda N, et al. An association study of bipolar mood disorder (type I) with the 5-HTTLPR serotonin transporter polymorphism in a human population isolate from Colombia. Neurosci Lett 2000;292:199-202.

10. Tirado MC. Estudio de la isonimia en la población de El Santuario. Medellín: Universidad de Antioquia; 1987.

11. Cavalli Sforza LL, Bodmer WF. Genética de las poblaciones humanas. Barcelona: Ediciones Omega; 1981.

12. Crow JF, Mange AP. Measurement of inbreeding from the frequency of marriages between persons of the same surname. Eugen Q 1965;12:199-203.

13. Pettener D, Pastor S, Tarazona-Santos E. Surnames and genetic structure of a high-altitude Quechua community from the Ichu River Valley, Peruvian Central Andes. Hum Biol 1998;70:865-87.

14. Wright S. The genetical structure of populations. Ann Eugen 1951;15:323-54.

15. Fisher RA. The relation between the number of species and the number of individuals in a random sample of animal population. J Anim Ecol 1943;12:42-58.

16. Morton NE. Kinship and population structure. En: Morton NE, editor. Genetic structure of populations. Honolulu: University Press of Hawaii; 1973. p.66-71.

17. Rodríguez-Larralde A, Formica G, Scapoli C, Beretta M, Mamolini E, Barrai I. Microevolution in Perugia: Isonymy 1890-1900. Ann Hum Biol 1993;20: 261-74.

18. Rodríguez-Larralde A. Distribución de los apellidos y su uso en la estimación de aislamiento y sedentarismo en municipios del Estado Lara, Venezuela. Acta Científica Venezolana 1990;41:163-70.

19. Bouchar G, De Braekeleer M. Historie d'un Génôme: Population et Génetique dans L'est du Québec. Quebec: Presses de L'université du Québec. Sillery; 1991.

20. Felsenstein, J. PHYLIP (Phylogeny Inference Package). Version 3.57c. Washington D.C.: University of Washington; 1995. 
21. Carvajal L, Ophoff R, Service S, Hartiala J, Molina J, Leon $\mathbf{P}$, et al. Genetic demographic of Antioquia (Colombia) and the Central Valley of Costa Rica. Hum Genet 2003;112:534-41.

22. Service S, DeYoung J, Karayiorgou M, Louw Roos J, Pretorious H, Bedoya G, et al. Magnitude and distribution of linkage disequilibrium in population isolates and implications for genome-wide association studies. Nat Genet 2006;38:556-60.

23. Immel UD, Krawczak M, Udolph J, Richter A, Rodig $\mathbf{H}$, Kleiber M, et al. Y-chromosomal STR haplotype analysis reveals surname-associated strata in the East-German population. Eur J Hum Genet 2006; 14:577-82

24. Alzate JM. Aranzazu: su historia y sus valores. Manizales: Instituto Caldense de Cultura; 2000.
25. Hoyos-Körbel PF. Café. Caminos de herradura y el poblamiento de Caldas. Bogotá: Tercer Mundo Editores; 2001.

26. López-Montes JF. Historia de Aranzazu. Medellín: Bell; 1960.

27. Jobling MA. In the name of the father: surnames and genetics. Trends Genet 2001;17:353-7.

28. Zei G, Lisa A, Fiorani O, Magri C, Quintana-Murci L, Semino $O$, et al. From surnames to the history of $Y$ chromosomes: the Sardinian population as a paradigm. Eur J Hum Genet 2003;11:802-7.

29. King TE, Ballereau SJ, Schurer KE, Jobling MA. Genetic signatures of coancestry within surnames. Curr Biol 2006;16:384-8.

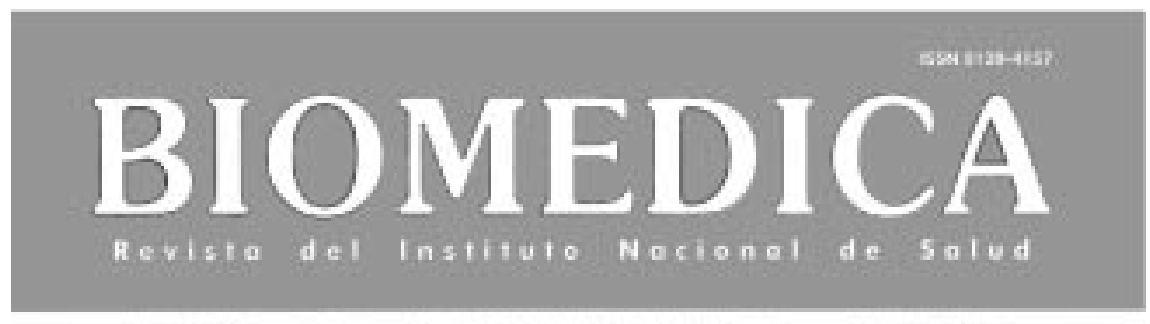

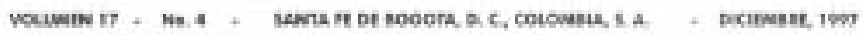

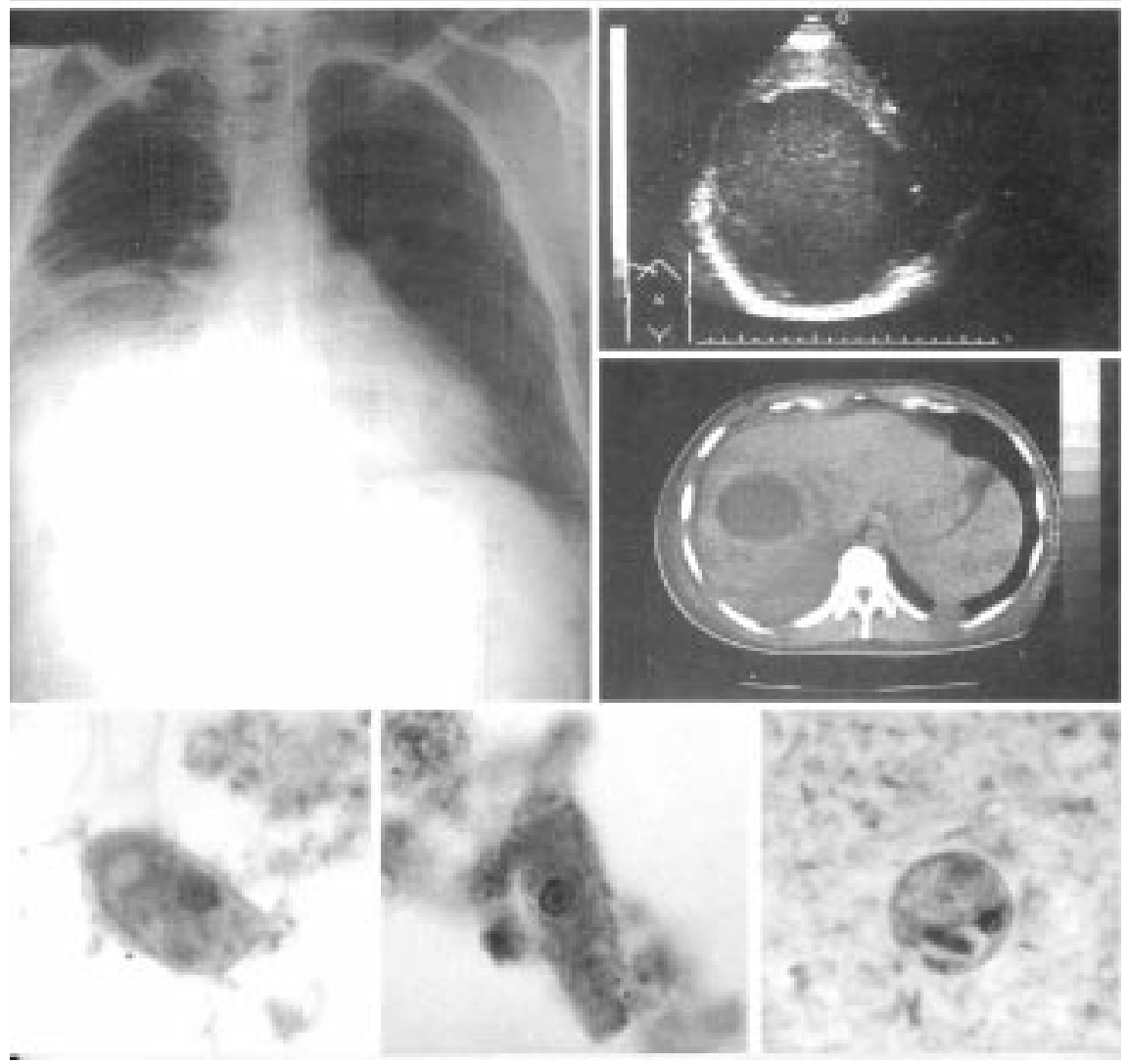

